\title{
VLBI OBSERVATIONS OF WR STARS
}

\author{
M. FELLI, M.MASSI \\ Osservatorio Astrofisico di Arcetri, Florence, Italy
}

\begin{abstract}
We present the first VLBI radio observations of two WR stars (WR140 and WR146) and of two early type stars (HD167971 and $\theta^{1}$ ORI A). The derived size and brightness temperature suggest that in all cases the radio emission originates from two distinct components: a steady weak emission due to the ionized wind of the primary and a smaller diameter brighter non-thermal component, probably associated to a small region within the ionized envelope of the star.
\end{abstract}

\section{Introduction}

The radio emission from WR and early type stars can either be thermal, i.e. it originates from the free-free emission of the envelope created by the mass loss and ionized by the stellar uv photons, or non-thermal. In the last case the emission mechanism is not yet fully understood and several alternatives have been proposed. Sources exhibiting both types of emission have also been found.

The radio data up to now came almost entirely from single dish or aperture synthesis observations, which only in few cases were able to resolve the thermal wind structure. In fact, for a typical mass loss of a WR star of $410^{-5} \mathrm{M}_{\odot} \mathrm{y}^{-1}$, a wind terminal velocity of $10^{3} \mathrm{Km} \mathrm{s}^{-1}$ and a distance of $1 \mathrm{kpc}$ the expected size at $6 \mathrm{~cm}$ is $\sim 215$ mas, which can barely be resolved with the VLA in the A configuration. Only in these sources the low brightness temperature found $\left(<10^{4} \mathrm{~K}\right)$ are consistent with a thermal interpretation.

For the unresolved sources the distinction between the two types of emission comes from the radio spectral index (a spectral index of 0.6 is expected for a thermal wind expanding at constant velocity, Panagia and Felli, 1975), or from the variability of the radio emission. However, neither of the two methods is known to be unambiguous. For instance the "bona fide" thermal wind of P Cygni is known to vary in the radio (Felli et al, 1985). Alternatively, if the mass loss derived from radio data is much greater than that derived from other diagnostics at optical and infrared wavelengths, the radio emission is suspected to be non-thermal.

Only VLBI observations are capable to reach the resolutions ( $\sim 10$ mas with the EVN and 1-2 mas with transatlantic baselines at $6 \mathrm{~cm}$ ) necessary to resolve the non-thermal components and to measure the source size and brightness temperature. In fact:

$$
T_{b} / \mathrm{K}=4.9^{*} 10^{7} S_{6 \mathrm{~cm}}(\mathrm{mJy})(\lambda / 6 \mathrm{~cm})^{2} /\left(\theta_{6 \mathrm{~cm}}(\mathrm{mas})\right)^{2} .
$$


For a $6 \mathrm{~cm}$ flux density of a few mJy (the present VLBI detection threshold) and a source size of a few mas the brightness temperature is $\sim 10^{6}-10^{7} \mathrm{~K}$.

However, due to the weakness of the radio emission, VLBI observations of WR stars and early type stars are still in the pioneering stage and, at present, mapping is very difficult. Only for a small number of stars a rather crude analysis of the fringe visibility curve is feasible.

We present VLBI observations at $6 \mathrm{~cm}$ with the European VLBI Network (EVN) of W146, as well as observations of WR140 (HD193793) which include also transatlantic baselines. This WR has also been monitored with the VLA and Westerbork in the last years and it is now in the rising part of its periodic radio emission light-curve.

These results are compared with recent EVN observations of HD167971 and $\theta^{1}$ ORI A, the last one observed also with transatlantic baselines.

All the above stars were suspected to be non-thermal radio emitters on the basis of the previously mentioned criteria and were selected from extended VLA surveys (Becker and White, 1985; Abbott et al, 1986; Churchwell at al, 1987; Bieging, Abbott and Churchwell, 1989 ) as the most favorable candidates for VLBI observations.

\section{VLBI and VLA results of individual stars}

\subsection{WR146}

WR146 is a WC4 star in the Cyg OB2 cluster (distance $2.0 \mathrm{kpc}$ ). Its radio emission was recently detected by Becker, Helfand and White (private communication) during a VLA survey of the galactic plane. It has a clear non-thermal spectral index $(\alpha=-1)$ and has remained bright on the few occasions that it was observed. On November 131989 the VLA $6 \mathrm{~cm}$ flux density was $32.4 \mathrm{mJy}$.

VLBI observations were carried on September 91989 with the five EVN antennas (Effelsberg, Jodrell, Westerbork, Medicina and Onsala, hereon E,J,W,M and O), with four integrations (13 min each) with Mark III tapes, mode A (i.e. total bandwith of $56 \mathrm{MHz}$ for maximum sensitivity). The source is clearly detected with a high $\mathrm{S} / \mathrm{N}$ (between 18 and 10 ) on all the four integrations with the shortest and most sensitive baseline (E-W). The mean flux density is $7 \pm 0.5 \mathrm{mJy}$, where the error represents the scatter around the mean.

A pure thermal wind explanation (already unlikely from the spectral index) can be ruled out from a comparison of VLA and EVN results. In fact, if all the $32.4 \mathrm{mJy}$ observed at the VLA came from a wind, the derived mass loss would be $1.210^{-4} \mathrm{M}_{\odot} \mathrm{y}^{-1}$, much greater than the mean value found for WR $\left(410^{-5} \mathrm{M}_{\odot} \mathrm{y}^{-1}\right)$. The implied angular size of such a wind would be $\sim 105$ mas and the expected flux density of a gaussian source of this size at the E-W baseline would be at least one order of magnitude lower than observed.

The most plausible hypothesis seems to be that of a wind plus a small diameter nonthermal component. For a mass loss of WR146 equal to the mean value of a WR the expected flux density of the thermal wind would be $\sim 5.5 \mathrm{mJy}$ and the size $\sim 43$ mas. The non-thermal flux density would then be $\sim 27 \mathrm{mJy}$. This value reduces to $7 \mathrm{mJy}$ on the $\mathrm{E}-\mathrm{W}$ baseline for a source size $\leq 30$ mas. The brightness temperature would be $\geq 1.5$ $10^{6} \mathrm{~K}$, clearly indicating the non-thermal nature of this component. The proposed two component configuration, which should be regarded only as indicative, has the advantage 
that the spectral index of the entire source is dominated by the non-thermal component, in agreement with the observations.

\subsection{WR140}

WR140 (HD193793) is a binary system composed by a WC7 and an O4-5, at a distance of $1.3 \mathrm{kpc}$. The orbital period is 7.94 years, with periastron of $2.35 \mathrm{AU}$ (1.8 mas) and apastron of $27.0 \mathrm{AU}$ (20.8 mas). Its near IR light-curve has a well defined periodicity with peak at periastron and period equal to the orbital one. For a complete description of the radio, X-ray and IR properties we refer to Williams et al (1990).

The radio emission is also known to vary with the same period, but shifted in phase. The peak occurs at $\phi \sim 0.8$. The emission appears to be quiescent for most of the orbital period, at a level of $1.5 \mathrm{mJy}$ at $6 \mathrm{~cm}$, and has a spectral index compatible with the canonical 0.6 value for a thermal wind. A thermal wind of $1.5 \mathrm{mJy}$ at $6 \mathrm{~cm}$ with a terminal velocity of $3000 \mathrm{Km}$ is produced by a mass loss of $510^{-5} \mathrm{M}_{\odot} \mathrm{y}^{-1}$ and has an angular size of $\sim 90$ mas. Clearly, the entire orbit of the 04-5 companion is within the the wind of the WR. At $\phi=0.3$ the emission begins slowly to increase and reaches a maximum as high as $25 \mathrm{mJy}$ at $6 \mathrm{~cm}$ at $\phi=0.8$. The spectral index is non-thermal at the peak. The next maximum should occur in 1991.

On December 1989 the 6 and $2 \mathrm{~cm}$ flux densities were 13.5 and $19.2 \mathrm{mJy}$, respectively (White and Becker, private communication). If we subtract from the observed fluxes the steady thermal wind component, the spectral index of the variable one is 0.23 , clearly changing to non-thermal values, although remaining positive. However, we remind that if the non-thermal component is produced within the WR wind, due to the wind optical depth the observed spectral index may differ noticeably from the intrinsic one. For instance, if the optical depth between the observer and the source due to the wind is $\tau_{\text {wind }}(6 \mathrm{~cm})=$ 1 , the intrinsic $2 \mathrm{~cm}-6 \mathrm{~cm}$ spectral index will be increased by 0.8 .

To study the relative contribution of the two components before the light-curve maximum we performed on March 71990 a MarkIII mode A $56 \mathrm{MHz}$ bandwidth VLBI experiment with the EVN plus the VLA in the phased array mode and the NRAO 140' (Green Bank). Since the observations were reduced only few weeks ago, the results are very preliminary. Software problems at $\mathrm{J}$ did not allow a proper recording of the signal. No fringes were detected with $\mathrm{O}$ due to the low $\mathrm{S} / \mathrm{N}$. The NRAO 140' recorded both polarization with $28 \mathrm{MHz}$ bandwidth each, leaving only one polarization for the correlation. At the EVN stations, where the high density was available, 16 scans were obtained, each of 13 minutes. Only one scan was available with transatlantic baselines. Fringes with high $\mathrm{S} / \mathrm{N}$ were detected on all scans on the E-W baseline and on 10 scans on the E-M baseline, the lost ones being due to instrumental failure. By using the rate and delay values of these two baselines, the $\mathrm{M}-\mathrm{W}$ baseline was refringed using a smaller search window: from -1 to -11 $\mathrm{mHz}$ for the rate and from 5 to $50 \mathrm{nsec}$ for SBD, narrower than the default values $(-200$ to $200 \mathrm{mHz}$ and -2000 to $2000 \mathrm{nsc}$, respectively).

For no intercontinental baseline there was a detection in the first fringe output based on the default values for delay and rate. The scans were refringed with a narrower search window derived from the calibrator's delay and rate. The results of all the baselines with the NRAO 140' (including that with the VLA) have been rejected as completely unreliable due to the high closure rate and delay for the available triangles. On the contrary, for the 
same arguments the results for the baselines with the VLA are acceptable. Since we have only one scan with the VLA it is hard to assess the reliability of these results.

To convert the observed fringe visibility into flux density we need to evaluate the b-factors. This is of no problem for the EVN baselines, but becomes difficult for the intercontinental baselines since the calibrator (3C418) is resolved. For the baselines with the VLA we have assumed a b-factor of 1.5, equal to the mean value on the EVN baselines.

The final observed flux densities on the EVN baselines are $17 \pm 1 \mathrm{mJy}$ for E-W, $9 \pm$ $3 \mathrm{mJy}$ for $\mathrm{E}-\mathrm{M}, 14 \pm 2 \mathrm{mJy}$ for $\mathrm{M}-\mathrm{W}$, with a mean value of $13.5 \mathrm{mJy}$. On the trasatlantic baselines the flux densities are $2.0 \pm 0.5 \mathrm{mJy}$ for E-VLA, $4 \pm 1$ for W-VLA and $4 \pm 1$ for M-VLA. For comparison, the VLA $6 \mathrm{~cm}$ flux density on March 31990 was $15 \mathrm{mJy}$ (White and Becker, private communication) and the Westerbork flux density on May 31990 was $16 \pm 1 \mathrm{mJy}$ (de Bruyn, private communication).

Using $13.5 \mathrm{mJy}$ as the indicative flux density of the variable component the derived angular size is 1.2 mas and the implied brightness temperature $4.910^{8} \mathrm{~K}$.

Even though the number of visibilities that we have is large, the gap between the EVN and the transatlantic baselines is so large that a two gaussian components fit to the visibilities (i.e. a $\sim 40$ mas wind with $1.5 \mathrm{mJy}$ and a smaller diameter source) gives a confidence factor undistinguishable from that of a single component. The existence of a 1.5 mJy wind (although formally equal to the difference between VLA and mean EVN fluxes) is within the noise of the VLBI data and its presence has to rely more securely on the previous history of the radio emission and on the spectral index of the steady component.

The separation of the two stars at the time of the VLBI observations (corresponding to a phase $\phi=0.62$ ) is $26.1 \mathrm{AU}$, or 20 mas. Given the parameters of the orbit, the projected separation is $\sim 17$ mas, greater than the source size.

We will tentatively associate the non-thermal component with the region of interaction between the wind of the $\mathrm{O}$ star and that of the WR. In fact, the absorption of the WR wind favours positions more distant than periastron. Even for moderate mass losses the WR wind may be completely opaque to the non-thermal component near periastron passage, regardeless of its intrinsic intensity. This optical depth effect can give an explanation to the disappearance of the non-thermal component after $\phi=0.8$ and the phase offset of the radio light-curve (Williams et al, 1990). In all the above it is assumed that the region of non-thermal emission is much closer to the $O$ star than to the WR, as expected form the lower intensity and velocity of the mass loss of the $\mathrm{O}$ star.

\section{$2.3 \mathrm{HD} 167971$}

HD167971 is thought to be a triple system with an O7.5 If primary. It was selected from the survey of Bieging et al (1989) for being classified as non-thermal on the basis of the spectral index $(\alpha=-0.7)$ and weak variability. The VLA flux density at $6 \mathrm{~cm}$ is $14 \mathrm{mJy}$.

The EVN observations of September 91989 could not have worst luck since E had problems with the field system and $M$ was down for repair. We report the result here for the benefit of documentation and in the hope that they may trigger further VLBI observations.

Only for the remaining most sensitive baseline, i.e $\mathrm{J}-\mathrm{W}$, we have a $\mathrm{S} / \mathrm{N} \sim 5.5$ and fringe and delay rate consistent with that of the calibrator. The derived flux density is 10 $\pm 2 \mathrm{mJy}$. Comparison with the VLA flux implies a source size of 6 mas and a brightness 
temperature of $210^{7} \mathrm{~K}$. The VLBI experiment confirms the non-thermal nature of the radio emission, but more observations with transatlantic baselines are needed to get a better description of this source.

\section{$2.4 \theta^{1}$ ORI A}

$\theta^{1}$ ORI A is one of the four Trapezium stars in the Orion Nebula. It has a spectral type B0.5 and hence it is much less luminous and emits a lower flux of ionizing photon than the brightest member $\theta^{1}$ ORI $C$, which is the main responsible for the ionization of the nebula.

Its radio emission was detected during VLA studies of the Orion Nebula (Gary, Moran and Reid, 1985; Churchwell et al, 1897) and re-observed in several other occasions. Its flux density can vary between $2 \mathrm{mJy}$ and $73 \mathrm{mJy}$ at $2 \mathrm{~cm}$. The spectral index, when measured, is $\sim 0.0$. For a complete description of the radio properties of the star we refer to Felli, Massi and Churchwell (1989).

The star is known to be a close binary system with a period of 65.43 days. The maximum and minimum separation between the primary and the secondary (presumed to be a TTauri) are 0.4 and 2.73 mas.

$6 \mathrm{~cm}$ EVN observations on September 281987 were able to detect the star and to establish that all the VLA flux density (14.8 $\mathrm{mJy}$ at that epoch) was contained in the VLBI structures, with an upper limit of 4 mas to the source size and a lower limit of $410^{7}$ $\mathrm{K}$ to the brigthness temperature.

We present here new VLBI observations with transatlantic baselines aimed to resolve the source structure. The experiment was performed on April 131989 and included the EVN antennas and the VLA. The VLA flux density was, at that epoch, $18 \pm 1 \mathrm{mJy}$. Similarly to our previous EVN run, the VLA flux density was equal to the visibility on the EVN baseline. The only transatlantic baseline for which we have a meaningful detection is E-VLA, which gives a flux density of $2.2 \pm 0.6 \mathrm{mJy}$. Also in this case we had to search in a smaller window of $\pm 5 \mathrm{mHz}$ in fringe rate and of $\pm 50 \mathrm{nsec}$ in SB delay.

A gaussian fit to the visibilities gives a source size of 1.3 mas and a brightness temperature of $610^{8} \mathrm{~K}$. Any steady extended thermal component, if present, must have a flux density $\leq 1 \mathrm{mJy}$.

With our VLA data base on Orion and with observations found in the literature we tried to see if there is any periodicity in the variable emission with a period similar to that of the binary. The data are rather scattered, partly for instrumental reasons since they come from VLA observations with different configurations and/or frequencies and, consequently, with different resolutions. However, they indicate, on the average, an increase of about a factor 3 with respect to the mean value $(12 \mathrm{mJy})$ at $\phi=0.85$, not dissimilar to what found for WR140.

On the last VLBI run the binary phase was 0.52 , and the separation of the two components was maximum. The fact that the radio source size is less than this separation suggests that the non-thermal source may be associated with one of the two stars. Following arguments similar to those used for WR140, we believe this is associated with the secondary and reaches maximum slightly before periastron.

In fact, early type stars are often surrounded by ionized envelopes produced by mass loss, even though less intense than for WR. For a B0.5 the expected mean mass loss is 
$\sim 10^{-8} \mathrm{M}_{\odot} \mathrm{y}^{-1}$. The expected flux density from this thermal source would be $\sim 0.002$ $\mathrm{mJy}$, clearly below detectability. However, the source size of this wind would be 0.9 mas, greater than the minimum separation of the two stars. Optical depth effects of this wind could be important when the secondary passes inside the primary wind and, similarly to WR140, make it more likely that the non-thermal radio emission is closer to the secondary. A model radio light-curve obtained taking into account the optical depth effects of the B0.5 wind and assuming an emissivity of the non-thermal component proportional to $\mathrm{r}^{-2}$ gives a satisfactory fit of the observations.

\section{Conclusions}

We have presented VLBI observations of WR and early type stars which establish the size and surface brightness of the non-thermal variable component. This is usually much smaller that the maximum binary separation.

The observed radio light-curves indicate a periodicity equal to the orbital period, suggesting a possible association between the non-thermal component and the secondary. Optical depth effects of the primary wind may strongly influence the observed intensity and spectral index and be the main responsible for the shape of the light-curve.

An other WR which shows two separate thermal and non-thermal components is WR147 (Moran et al, 1989), a binary system with much greater separation (1100 AU, the two components are clearly separated) and a much longer period $\left(10^{3} \mathrm{y}\right.$, no information on the radio variability or periodicity is available).

The common binary nature of all these systems seems to suggest that $\theta^{1}$ ORI A, WR140 and WR147 are scaled up version of the same phenomenon, i.e. they are composed by a steady component due to the thermal wind and a variable non-thermal one due to the interaction between the mass loss of the primary and the weaker wind of the secondary.

More sensitive VLBI observations are needed to set the position of the non-thermal component with respect to the faint elusive extended thermal wind.

\section{References}

Abbott,D.C., Bieging,J.H., Churchwell,E., 1986, Ap.J., 303, 236.

Becker, R.H., White, R.L., 1985, Ap.J., 297, 649.

Bieging,J.H., Abbott,D.C., Churchwell,E., 1989, Ap.J., 340, 518.

Churchwell,E., Felli,M., Wood,D.O.S., Massi,M., Ap. J., 321, 516.

Felli, M., Stanga,R., Oliva,E., Panagia, N.. 1985, Astron. Astrophys 151, 27.

Felli,M., Massi,M., Churchwell,E., 1989, Astron. Astrophys., 217, 179.

Garay,G., Moran, J.M., Reid,M.J., 1985, in Radio Stars, R.M. Hjellming and D.M. Gibson eds, 131 .

Moran,J.P., Davis,R.J., Bode,M.F., Taylor,A.R., Spencer,R.E., Argue,A.N., Irwin,M.J., Shanklin,J.D., 1989, Nature, 340, 449.

Panagia, N., Felli, M., 1975, Astron. Astrophys., 39, 1.

Williams,P.M., van der Hucht,K.A., Pollock,A.M.T., Florkowski,D.R., van der Woerd,H., Wamsteker,W.M., Mon.Not.R.Astr.Soc., 1990, 243, 662. 


\section{DISCUSSION}

Owocki: All the objects you mentioned were binaries. Is it not possible to also use this technique to interpret proporties of radio emission from single stars?

Felli: The radio stars included in the VLBI program were selected only for having the largest flux density and being unresolved with the VLA. The fact that they are binary systems is a pure coincidence. VLBI techniques can be used for any radio star (single or binary) provided that there are radio components with sufficient flux density at resolutions of a few $m J y$.

Cassinelli: Can you use your brightness temperature to estimate the magnetic field associated region, where the radio flux is coming from?

Felli: We have not yet started to model which is the source of emission, so, I can not give you an answer.

Montmerle: MERLIN has resolved AS 431 into two components, one thermal and the other non-thermal. Do you see any indication of this in your stars?

Felli: The main goal of these VLBI observations was to establish if there were small diameter non-thermal components. We tried a map of WR140 with the limited visibilities available. The first map clearly indicates the presence of two distinct components. However the UV coverage and the sensitivity are too small to allow a proper mapping of the extended $1.5 \mathrm{~mJ} y$ thermal wind. New VLBI observations (by us and the van der Hucht group) with better UV coverage are planned for WR140. 


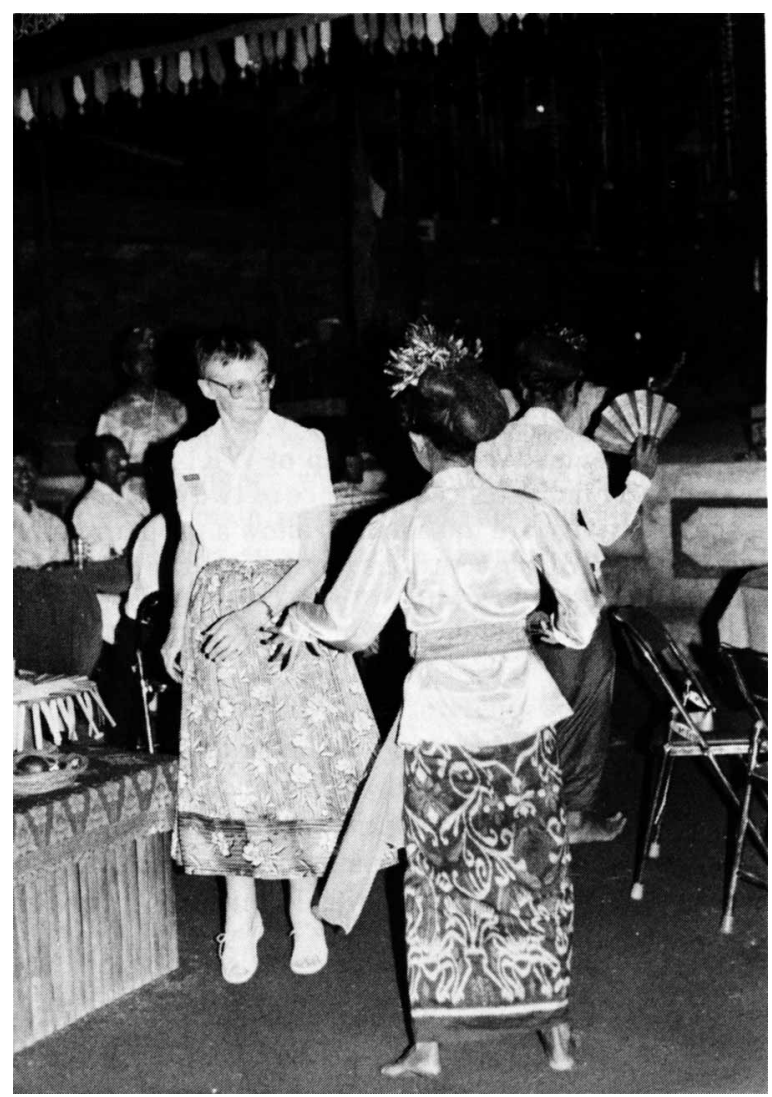

Anne Underhill, Balinese dancer 